\title{
A Simple but Dramatic Technical Improvement in the Diff Quik Stain Protocol Used for Preparing Specimens for Sperm Morphology Evaluation (Improved Diff Quik Stain Protocol for Preparation of Sperm Morphology Evaluation Specimens)
}

\author{
F. Aono, K. Ochiai, T. Ueno, T. Okubo, and S. Teramoto
}

Background: Understanding an accurate semen analysis result is crucial for planning a couple's appropriate infertility treatment. In particular, assessment of sperm morphology is difficult but critically important to accurately ascertain. The Diff Quik stain protocol, which was introduced in the WHO semen analysis manual (5th edition), is simple and rapid, but the staining patterns are highly variable.

Main Questions: Does the Diff Quik stain protocol create a homogeneous stained sample, and can reproducibility be improved?

Experimental Design: We compared semen volumes of 3, 5, and $10 \mu \mathrm{L}$ respectively for smear samples; duration in fixation solution of 10, 30, and $60 \mathrm{~s}$ in the stain solution II; and running water versus a tiny $150 \mu \mathrm{L}$ volume of water, to eliminate the excess stain solution II.

Main Results: We obtained the most consistent results with a $3 \mu \mathrm{L}$ semen smear sample volume, $30 \mathrm{~s}$ of duration in fixation solution, $30 \mathrm{~s}$ in the stain solution I, $60 \mathrm{~s}$ duration in staining solution II, and $150 \mu \mathrm{L}$ of water for eliminating the excess stain solution II. We applied this improvement in the Diff Quik stain protocol to mouse Macaque monkey and human semen samples with the same consistent results.

Conclusions: We have established an improvement of the Diff Quik stain protocol for evaluation of sperm morphology, and this new protocol has made it possible to obtain reliable, and repeatable results with uniform consistency for precise evaluation of sperm morphology.

F. Aono $(\varangle) \cdot$ K. Ochiai $\cdot$ T. Ueno $\cdot$ T. Okubo $\cdot$ S. Teramoto

Natural ART Clinic Nihombashi, Tokyo, Japan

e-mail: f-aono@yumeclinic.net 